\title{
Simulations to compare efficacies of tetravalent dengue vaccines and mosquito vector control
}

\author{
U. THAVARA ${ }^{1}$, A. TAWATSIN ${ }^{1}$ AND Y. NAGAO ${ }^{2 *}$ \\ ${ }^{1}$ National Institute of Health, Ministry of Public Health, Nonthaburi, Thailand \\ ${ }^{2}$ Onoda Hospital, Haramachi-ku, Minami-soma city, Fukushima, Japan
}

Received 1 November 2012; Final revision 3 June 2013; Accepted 10 July 2013;

first published online 8 August 2013

\section{SUMMARY}

Infection with dengue, the most prevalent mosquito-borne virus, manifests as dengue fever (DF) or the more fatal dengue haemorrhagic fever (DHF). DHF occurs mainly when an individual who has acquired antibodies to one serotype is inoculated with another serotype. It was reported that mosquito control may have increased the incidence of DF and DHF due to age-dependency in manifesting these illnesses or an immunological mechanism. Tetravalent dengue vaccine is currently being tested in clinical trials. However, seroconversions to all four serotypes were achieved only after three doses. Therefore, vaccines may predispose vaccinees to the risk of developing DHF in future infections. This study employed an individual-based computer simulation, to emulate mosquito control and vaccination, incorporating seroconversion rates reported from actual clinical trials. It was found that mosquito control alone would have increased incidence of DF and DHF in areas of high mosquito density. A vaccination programme with very high coverage, even with a vaccine of suboptimal seroconversion rates, attenuated possible surges in the incidence of DF and DHF which would have been caused by insufficient reduction in mosquito abundance. DHF cases attributable to vaccine-derived enhancement were fewer than DHF cases prevented by a vaccine with considerably high (although not perfect) seroconversion rates. These predictions may justify vaccination programmes, at least in areas of high mosquito abundance. In such areas, mosquito control programmes should be conducted only after the vaccination programme with a high coverage has been initiated.

Key words: Dengue haemorrhagic fever, mathematical modelling, vaccine safety, vaccines, vector control.

\section{INTRODUCTION}

Infection by dengue virus causes a wide variety of illnesses ranging from rarely lethal dengue fever (DF) to dengue haemorrhagic fever (DHF), the latter of which results in a case-fatality rate of more than $10 \%$ unless

\footnotetext{
* Author for correspondence: Dr Y. Nagao, Onoda Hospital, Haramachi-ku, Minami-soma city, Fukushima, Japan. (Email: in_the_pacific214@yahoo.co.jp)
}

adequately treated $[1,2]$. With no approved vaccine available, reduction of vector mosquitoes has been regarded as the only means of controlling dengue. However, whether mosquito control has actually reduced dengue illnesses is under debate. Reduction of vector mosquitoes results in a higher average age at which primary infections occur [3]. Since primary infections often are asymptomatic in children but manifest as illness in adults [4, 5], this rise in host age appears to have increased the incidence of DF 
in Singapore [6]. As the average age of dengue infection is increasing in many countries [7], this mechanism, known as 'endemic stability' [8], may explain the rising incidence of DF in some countries.

The unique immunological aetiology of DHF may have led to a similar unwanted consequence as well [9]. DHF is known to occur in a secondary infection more frequently than in a primary infection, due to immunological mechanisms including antibodydependent enhancement (ADE) [10]. On the other hand, many of the individuals in areas of high vector mosquito abundance would be infected by, and acquire immunity against, multiple serotypes while they are clinically protected by this cross-immunity [11]. Consequently, these individuals develop resistance to DHF unknowingly, since those infected by more than one serotype rarely manifest DHF [12]. As mosquito abundance decreases, an increasing number of individuals would experience secondary infections after the protective cross-immunity has waned, and the incidence of DHF would increase. Consistent with this hypothesis, incidence of DHF was in a negative relationship with mosquito abundance in Thailand $[9,13]$.

Whether these seemingly paradoxical hypotheses are true or not, the concern that insufficient mosquito control may increase the incidence of DF and DHF underscores the importance of understanding the effect of mosquito control and future vaccination on the target population. In effect, two tetravalent live vaccines were developed and tested in clinical trials: a classical live-attenuated vaccine from the Walter Reed Army Institute of Research (WRAIR) [14] and a chimeric yellow fever-dengue composite vaccine (ChimeriVax or CYD) from Sanofi-Pasteur [15]. Of these, only CYD remained in phase II $b$ trials [16]. However, dengue vaccines harbour a unique concern: enhancing antibodies may be induced by the vaccine itself $[17,18]$. In particular, a classical live-attenuated vaccine, which induces immune responses similar to those induced by wild-type viruses [19], may predispose a vaccinee to the risk of enhancement upon a subsequent infection. The risk of inducing enhancement by CYD, which is a yellow fever vaccine framework inserted with $p r M$ and $E$ genes from dengue viruses, is regarded as being less than for classical live-attenuated vaccine [19]. However, 'only phase IV trials and post-marketing surveillance will provide a definitive answer as to whether ADE constitutes a risk for vaccinees' [15]. Therefore, seroconversion to all four serotypes is regarded as a prerequisite for a tetravalent dengue vaccine. By contrast, in clinical trials conducted to date, live tetravalent vaccines induced antibodies to less than four serotypes in a considerably large proportion of vaccinees, even after two consecutive injections [20, 21]. Although seroconversions to all four serotypes were achieved after three injections, such a three-dose regimen of a live vaccine is unprecedented. Furthermore, administering all three doses to all vaccinees may be difficult to achieve in developing countries.

It is recognized that mathematical models are useful to predict the population-level effects of dengue vaccine [22]. Alternatively, the present study used an individual-based model, based upon results from clinical trials. Mathematical models creates a set of differential equations. In contrast, individual-based models create a large number of human individuals in the computer's memory, and observes their behaviour [23]. Diverse scenarios, regarding seroconversion rates of dengue vaccine and vaccination coverage were compared. In addition, the incidence of DHF attributable to ADE derived from prior vaccination was estimated. Finally, the optimal strategy for dengue control is discussed.

\section{MATERIALS AND METHODS}

\section{Assumptions for protective and enhancing antibodies}

While the titre of antibodies against dengue virus is high, the protective role of antibodies is dominant: however, as the titre wanes, antibodies enhance development of DHF [24]. It is not known whether protective antibodies and enhancing antibodies are physically separable (Fig. 1a) ([25] and E. Konishi et al., personal communication), or whether the same antibodies switch from protection to enhancement as their titres wane - in other words, virtual enhancing antibody (Fig. 1b) [26]. However, these two hypotheses converge into an identical software coding if the first hypothesis (Fig. 1a) surmises that the enhancing antibody persists life-long and if the second hypothesis (Fig. 1b) assumes that antibody induced by viral inoculation exerts life-long enhancement. This framework enabled the distinction between enhancing antibodies induced by prior wild-type infection and those attributable to vaccine.

An inoculation with a wild-type virus induces protective antibodies specific to that serotype. On the other hand, an individual inoculated with a tetravalent live vaccine acquires protective antibodies specific only to the serotype(s) to which s/he seroconverted. 

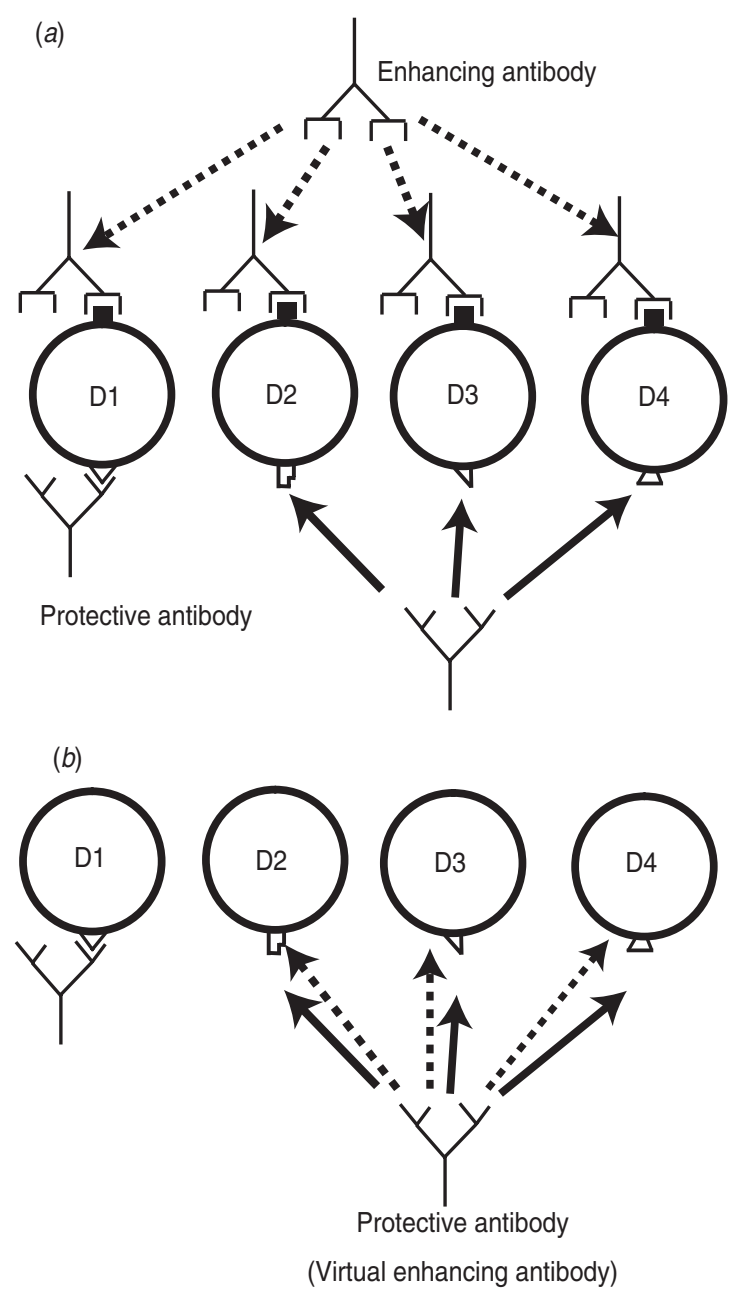

Fig. 1. Two hypotheses regarding antibodies to dengue viruses. (a) Enhancing antibodies and protective antibodies are different. Enhancing antibodies react with all virus serotypes (broken arrows). In contrast, protective antibodies are specific to a serotype (D1 in this figure), but exert transient cross-reactive protection against other serotypes (solid arrows). (b) The same antibodies may play different roles in protection and enhancement, depending on their titres. Antibodies (specific to D1 in this figure) exert protection against other serotypes when their titres are high (solid arrows). As the titre wanes, these antibodies act as enhancing antibodies (broken arrows). D1, D2, D3, and D4 represent dengue virus serotypes $1,2,3$, and 4 , respectively.

The protective antibodies are assumed to exert lifelong serotype-specific protection, as well as transient cross-serotype protection for a duration of $C$ years from the latest inoculation. Sabin [11] observed volunteers who were inoculated sequentially with two different strains of dengue virus, with the interval between the inoculations being maximally 9 months. It was found that cross-protection against severe illness still persisted at least after this interval. In addition, vaccination with yellow fever-dengue 2 chimeric vaccine induced cross-serotype protection that lasted $>1$ year [27]. Furthermore, it was reported that secondary infections resulted in DHF, DF, and asymptomatic infections at $2 \cdot 6,1 \cdot 9$, and $1 \cdot 6$ years after the primary infections, respectively [28]. These observations suggested that cross-serotype protection against DHF may last for $>1$ year. Therefore, $C$ was assumed to follow a normally distributed probability distribution function (PDF) with a mean equal to 2 years (Table 1). An individual inoculated with either wildtype or vaccine acquires enhancing antibodies, which persist throughout that individual's life [29, 30].

\section{Assumptions for DF and DHF}

Individual-based model simulation software (detailed in Protocol S1 of reference [13]) was modified to describe immunological behaviour of the host (Fig. 2a). When a naive individual is inoculated by wild-type dengue virus, s/he transitions to the crossprotected state. In the course of this transition, s/he may develop DF with an age-dependent probability defined in Figure $2 b$ (constructed based upon reference [5] and P. G. Coleman, personal communication), and DHF with a fixed small probability of $0 \cdot 2 \%$ [12]. If an individual in the cross-protected state is inoculated with a virus serotype, s/he acquires antibodies specific to this serotype and remains in the cross-protected state. $C$ years after the most recent inoculation, the individual moves to the expired crossprotection state. When an individual in this state is inoculated with a serotype to which s/he does not possess specific antibodies (i.e. unexperienced serotype), $\mathrm{s} /$ he may manifest DF. The individual may also develop DHF with an enhanced probability of $4 \%$ [12], if s/he already possesses enhancing antibodies. An individual who has seroconverted to ' $L$ ' serotype transitions to the completely immune state (Table 1). The transmissibility (or viraemia) may be enhanced ' $\mathrm{T}$ '-fold during manifestation of DHF [31] (Table 1).

\section{Basic and effective reproductive numbers}

In the mathematical models for dengue proposed so far, transmission intensity was often expressed as mosquito density $[32,33]$ or as basic reproductive number $\left(R_{0}\right)[9,34,35]$. In the present study, $R_{0}$ represents transmission intensity, since $R_{0}$ is proportional to vector abundance $[3,36,37]$. The range of $R_{0}$ was selected, considering previous estimates [34, 38, 39].

The immunological state of each individual was updated at discrete time-steps of 2 weeks' length, 
Table 1. Variable parameters given to simulations

\begin{tabular}{|c|c|c|c|}
\hline Definition & Symbol & Unit & $\begin{array}{l}\text { Values assumed } \\
\text { in simulations }\end{array}$ \\
\hline Cross-protective period & $C$ & Year & $\operatorname{PDF}($ mean $=2$, S.D. $=0 \cdot 5)$ \\
\hline $\begin{array}{l}\text { Number of serotypes necessary to confer } \\
\text { complete resistance to DHF }\end{array}$ & $L$ & Dimensionless & Randomly selected from 2,3 or 4 \\
\hline $\begin{array}{l}\text { Enhancement of transmissibility during } \\
\text { manifestation of DHF }\end{array}$ & $T$ & Dimensionless & $\operatorname{PDF}($ mean $=10$, s.D. $=2 \cdot 5)$ \\
\hline Basic reproductive number & $R_{0}$ & Dimensionless & Fixed* (range 1-20) \\
\hline Inhomogeneous mixing & $I$ & Dimensionless & Fixed* (range $0-1)$ \\
\hline Vaccination coverage & $V$ & $\%$ & Fixed* (range $0-100)$ \\
\hline Seasonality & $S$ & Dimensionless & Fixed* (range $0-1)$ \\
\hline Total fertility rate & TFR & Dimensionless & Fixed* (range $0-1$ ) \\
\hline
\end{tabular}

PDF, Probability distribution function with normal distribution; s.D., standard deviation.

* Fixed, a fixed value was input into each simulation to examine the effect of the parameter on the simulation result.

which approximates the sum of intrinsic incubation and infectious periods [11, 31, 40]. The risk of being infected by a serotype (force of infection or viral inoculation rate) at the $i$ th time-step $\left(F_{i}\right)$ was obtained as:

$F_{i}=R_{0, i-1} \times U_{i-1} / N_{i-1}$,

where $R_{0, i-1}, U_{i-1}$ and $N_{i-1}$ represent basic reproductive number, viraemic load and population size in the $(i-1)$ th time-step, respectively. Here, the viraemic load at the $i$ th time step $\left(U_{i}\right)$ is defined as:

$U_{i}=\mathrm{DF}_{i}+\left(T \times \mathrm{DHF}_{i}\right)$,

where $\mathrm{DF}_{i}$ and $\mathrm{DHF}_{i}$ denote the number of patients with DF and DHF, respectively. $T$ is the abovementioned enhancement in transmissibility (Table 1). Effective reproductive number, which is defined as the secondary infectious cases originating from a primary infectious case, was estimated for each time step to be compared with $R_{0}$ given to the simulation.

\section{Estimation of vaccine seroconversion rates}

To exclude the effect of natural infections, the results of clinical trials conducted in non-endemic areas were used in the present study (Table 2). Two trials adopted a regimen of $0-3 \cdot 5-12$ months [20,21]. However, the short first interval resulted in trans-serotype interference $[15,21]$. Therefore, the present study assumed 0-6-12 months interval (Table 3). The results in adult vaccinees were applied to paediatric vaccinations. All the trials in Table 2 defined a $50 \%$ plaque reduction neutralization titre $\left(\mathrm{PRNT}_{50}\right)$ of $1: 10$ as the cut-off for seropositivity. This titre had been considered as the surrogate for maximal protection, as in Japanese encephalitis [41]. The clinical studies reported the seroconversion rate after the first dose $\left(R_{i, 1 \%}\right)$, that after the second dose $\left(R_{i, 1+2 \%}\right)$ and that after the third dose $\left(R_{i, 1+2+3 \%}\right)$ for each serotype $i(i=1,2,3$, or 4$)$. From these values, the seroconversion rate achieved solely by the second dose $\left(R_{i, 2}\right)$ was estimated as:

$R_{i, 2}=100 \times\left(R_{i, 1+2}-R_{i, 1}\right) /\left(100-R_{i}, 1\right)$,

Similarly, the seroconversion rate achieved solely by the third dose $\left(R_{i, 3}\right)$ was estimated as:

$R_{i, 3}=100 \times\left(R_{i, 1+2+3}-R_{i}, 1+2\right) /\left(100-R_{i}, 1+2\right)$,

Here, it was assumed that vaccinees that had seroconverted would not turn seronegative.

\section{Compromised protective seroconversion rates}

$R_{i, j}(i=1-4, j=1-3)$ represents the maximal achievable protective potencies. However, the efficacies reported in the clinical trial were low (i.e. 30.2\%) [16], indicating that 'protective' seroconversion rates for CYD may be much lower than $R_{i, j}$, which was measured based on a PRNT 50 of 1:10. Therefore, the present study conducted a sensitivity analysis by considering tetravalent vaccines with compromised protective potencies (Table 3). $\mathrm{CYD}_{40}$ and $\mathrm{CYD}_{20}$ were assumed to induce protective seroconversion rates of $40 \%$ and $20 \%$, respectively, compared to the seroconversion rates measured as $\mathrm{PRNT}_{50}$ of $1: 10 . \mathrm{CYD}_{100}$ induces protective seroconversion rates equal to those measured as $\mathrm{PRNT}_{50}$ of $1: 10$.

\section{Vaccine assumptions}

Enhancing antibodies derived from vaccine and those from wild-type virus were differentiated in the 

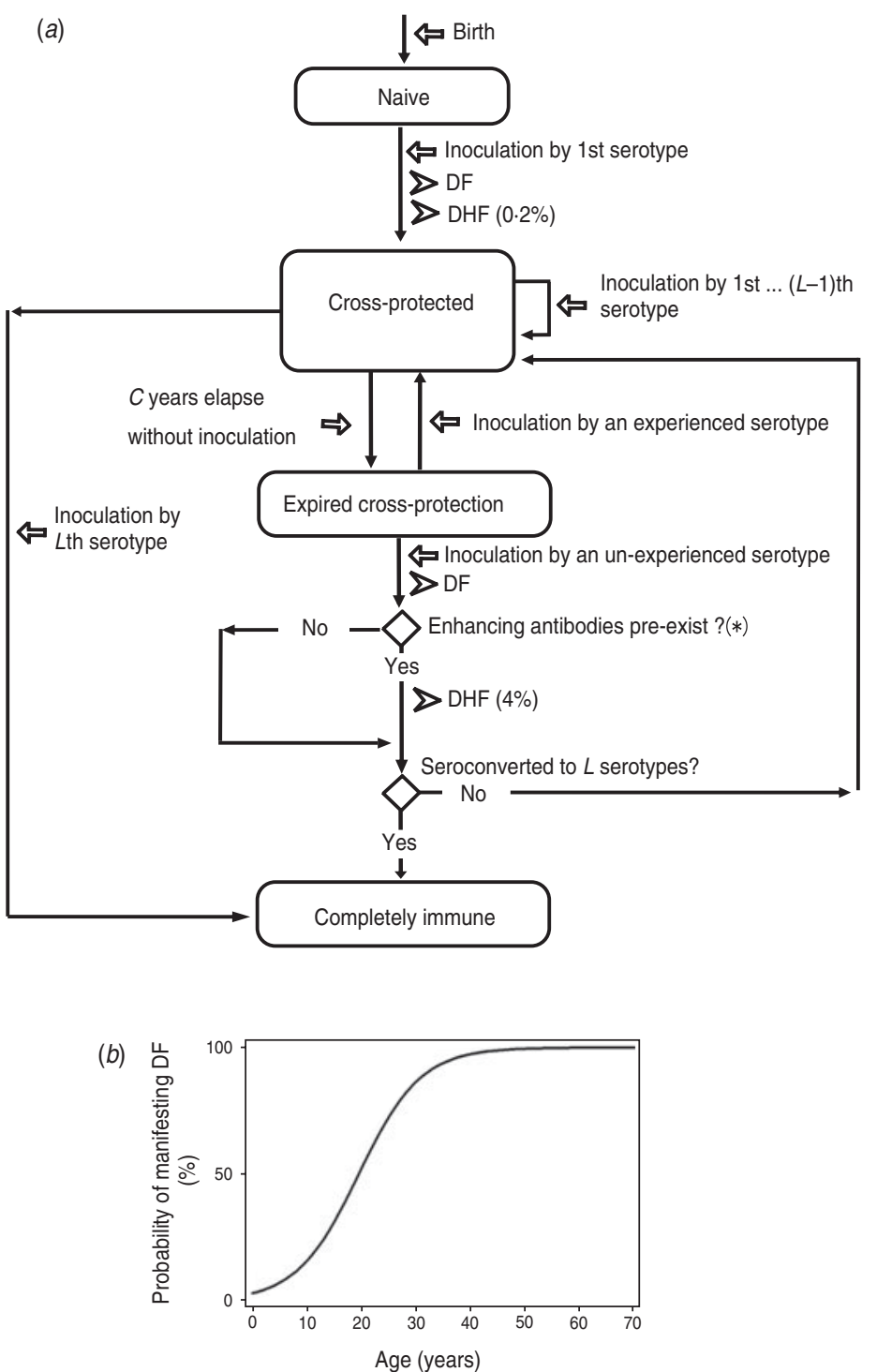

Fig. 2. Individual-based model for dengue infections. (a) Diagram of the transition between immunological states. Transition between immunological states (filled arrow) occurs as a result of either viral inoculation or expiration of time from the most recent inoculation (open arrow). During a state transition, the individual may manifest dengue fever (DF) or dengue haemorrhagic fever (DHF) (arrow head). * A modification of the model in reference [13] was made: the individual is predisposed to the risk of DHF only if enhancing antibodies pre-exist. (b) Age-dependent probability for an infection to manifest as DF in an individual who is not protected specifically or cross-reactively. The probability is expressed as: $100 /[1+1 / \exp (-3 \cdot 44+0 \cdot 177 \times$ age $)] \%$ ([5] and P. G. Coleman, personal communication).

software. Inoculation by a vaccine will not manifest as clinical illness or lead to development of transmissible viraemia. Since CYD tetravalent vaccine contains only two dengue-virus peptides (E and prM), this vaccine may not confer efficient cross-protection. Consistent with this assumption, the efficacy of CYD measured within 2 years after vaccination was only $30 \%$ [16]. Therefore, this study adopted an unfavourable scenario for CYD, assuming that CYD does not induce cross-serotype protection.

\section{Emulation of vaccination programme}

A future vaccination programme was emulated. At the 100 th year in a 150 -year simulation, a vaccination programme was initiated. After this year, an attempt was made to vaccinate all children who reached age 2 years but only with a successful coverage of $V \%$. This age was selected because it was the youngest age tested in the clinical trials so far conducted. It was attempted to re-vaccinate the vaccinees at 6 months 
Table 2. Serotype-specific seroconversion rates (\%) of CYD tetravalent dengue vaccine based on a $P R N T_{50}$ of 1:10, obtained from clinical trials conducted in non-endemic areas

\begin{tabular}{|c|c|c|c|c|c|c|}
\hline & Age & $\begin{array}{l}\text { Time of } \\
\text { vaccination }\end{array}$ & D1 & D2 & D3 & D4 \\
\hline Ref. [20], Figure $4 a$ & $2-45$ & & & & & \\
\hline After 1st dose & & 0 month & 15 & 32 & 50 & 67 \\
\hline After 2nd dose & & $3 \cdot 5$ months & 50 & 75 & 72 & 87 \\
\hline After 3rd dose & & 12 months & 77 & 85 & 90 & 89 \\
\hline Ref. [21], Figure $2 a$ & $18-45$ & & & & & \\
\hline After 1st dose & & 0 month & 12 & 67 & 27 & 64 \\
\hline After 2nd dose & & 4 months & 70 & 93 & 73 & 87 \\
\hline After 3rd dose & & $12-15$ months & 100 & 100 & 100 & 100 \\
\hline Ref. [21], Figure $2 b$ & $18-45$ & & & & & \\
\hline After 1st dose & & 0 month & 30 & 72 & 33 & 88 \\
\hline After 2nd dose & & 8-11 months & 92 & 100 & 100 & 100 \\
\hline
\end{tabular}

D1, D2, D3, and D4 represent dengue virus serotypes 1, 2, 3, and 4, respectively.

Table 3. Dengue serotype-specific seroconversion rates $(\%)$ assumed in simulations

\begin{tabular}{|c|c|c|c|c|}
\hline & D1 & D2 & D3 & D4 \\
\hline \multicolumn{5}{|c|}{ Seroconversion rates from [21] Figure $2 a$} \\
\hline$R_{i, 1}$ & 30 & 72 & 33 & 88 \\
\hline$R_{i, 1+2}$ & 92 & 99 & 99 & 99 \\
\hline$R_{i, 1+2+3}$ & 100 & 100 & 100 & 100 \\
\hline \multicolumn{5}{|l|}{$\mathrm{CYD}_{100}$} \\
\hline$R_{i, 1}$ & 30 & 72 & 33 & 88 \\
\hline$R_{i, 2}$ & 89 & 96 & 99 & 92 \\
\hline$R_{i, 3}$ & 100 & 100 & 100 & 100 \\
\hline \multicolumn{5}{|l|}{$\mathrm{CYD}_{40}$} \\
\hline$R_{i, 1}$ & 12 & 29 & 13 & 35 \\
\hline$R_{i, 2}$ & 36 & 38 & 40 & 37 \\
\hline$R_{i, 3}$ & 40 & 40 & 40 & 40 \\
\hline \multicolumn{5}{|l|}{$\mathrm{CYD}_{20}$} \\
\hline$R_{i, 1}$ & 6 & 14 & 7 & 18 \\
\hline$R_{i, 2}$ & 18 & 19 & 20 & 18 \\
\hline$R_{i, 3}$ & 20 & 20 & 20 & 20 \\
\hline
\end{tabular}

$R_{i, 1}, R_{i, 1+2}$ and $R_{i, 1+2+3}$ denote the seroconversion rate for serotype $i$ after the first, second, and third doses, respectively. $R_{i, 2}$ and $R_{i, 3}$ represent the seroconversion rate achieved solely by the second and third doses, respectively. $R_{i, 2}$ and $R_{i, 3}$ of $\mathrm{CYD}_{100}$ were estimated using equations (3) and (4) in the main text, respectively. $R_{i, 1}, R_{i, 2}$, and $R_{i, 3}$ of $\mathrm{CYD}_{40}$ and those of $\mathrm{CYD}_{20}$ were $40 \%$ and $20 \%$ of the corresponding values for $\mathrm{CYD}_{100}$, respectively. $R_{I, 1}, R_{i 2}$ and $R_{i, 3}$ were input into the simulations. D1, D2, D3, and D4 represent dengue virus serotypes $1,2,3$, and 4 , respectively.

and 12 months after the first dose, but with a successful follow-up coverage of $V \%$, respectively. This was because $100 \%$ accomplishment of any vaccination programme, especially of three-dose regimen, is difficult to achieve in developing countries.

\section{Inhomogeneous mixing}

The degree of inhomogeneity in the mixing pattern was represented by $I$, where $I=0$ indicated completely homogeneous mixing and $I=1$ indicated maximally inhomogeneous mixing (see Supplementary online Appendix 1 and Table 1).

\section{Seasonality}

Since vector mosquito abundance fluctuates seasonally [42], seasonality is an important factor which should be considered in modelling a mosquito-borne disease [33]. Therefore, the present study expressed seasonality as a sinusoidal function of $R_{0}$, as follows:

$R_{0}(\theta)=\bar{R}_{0} \times[1-\cos (\theta) \times S]$,

where $\theta$ represents the phase of a time-step in a year $[0 \leqslant \theta \leqslant 2 \pi], R_{0}(\theta)$ indicates $R_{0}$ at this phase, and $\bar{R}_{0}$ expresses the average annual $R_{0} . S$ represents the strength of seasonality. $S=1$ indicates the strongest seasonality and $S=0$ represents no seasonality (Table 1).

\section{Age-specific birth rate and mortality rate}

Demographic data from Thailand were used as an example of a region heavily afflicted by dengue. Age-population structure of Thailand in 1960 was 
(a) Viral inoculation rate, $C Y D_{100}$

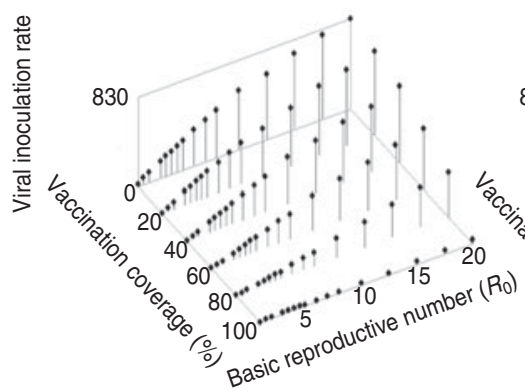

(d) DF incidence, $C Y D_{100}$

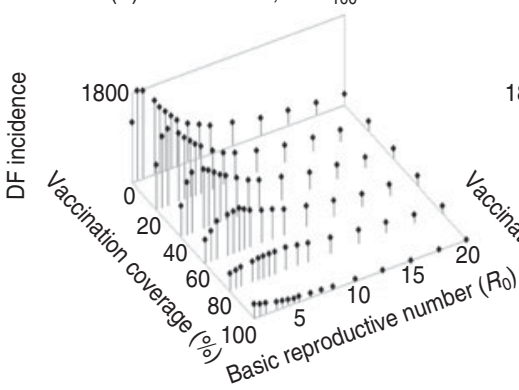

(g) DHF incidence, $\mathrm{CYD}_{100}$

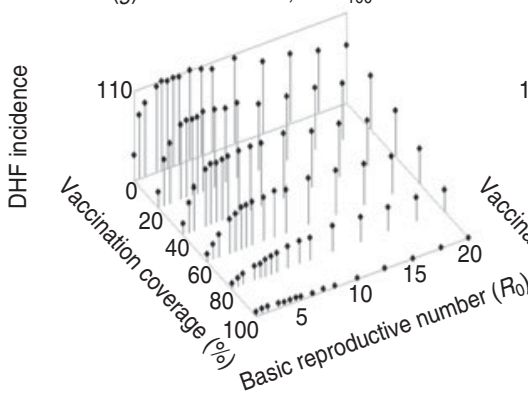

(j) Vaccine-derived DHF, CYD 100 (b) Viral inoculation rate, $\mathrm{CYD}_{40}$

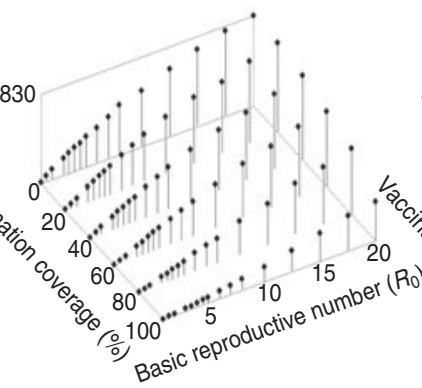

(c) Viral inoculation rate, $\mathrm{CYD}_{20}$

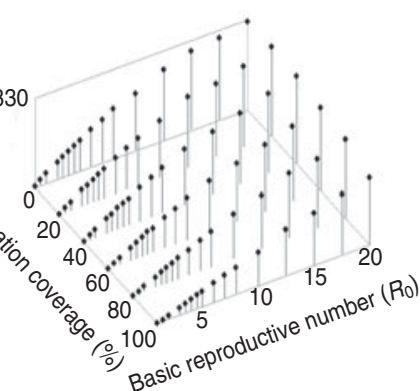

(e) DF incidence, $\mathrm{CYD}_{40}$

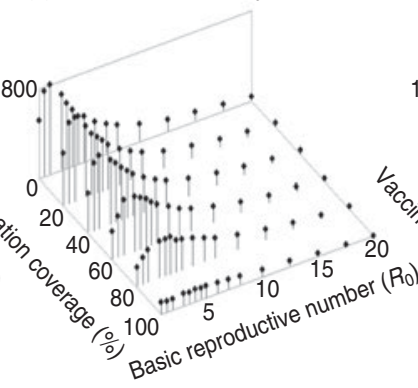

(f) DF incidence, $\mathrm{CYD}_{20}$
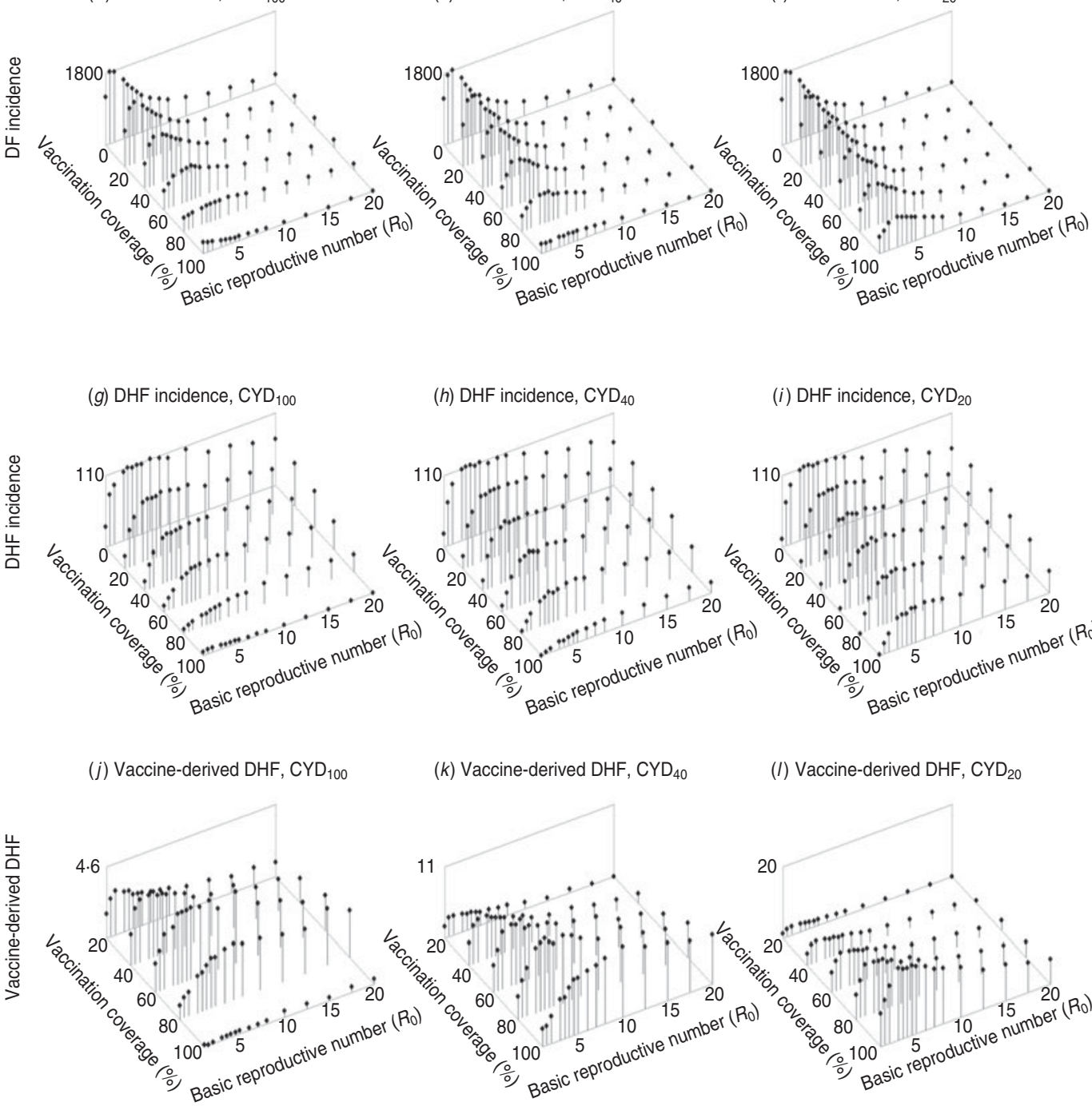

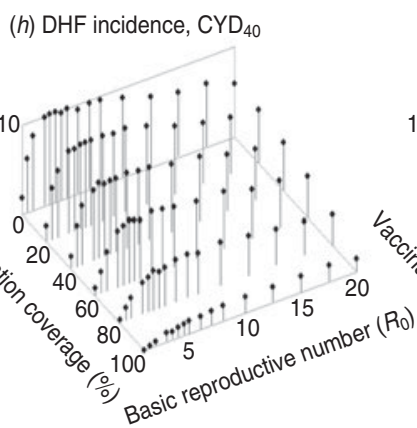

(k) Vaccine-derived DHF, $\mathrm{CYD}_{40}$

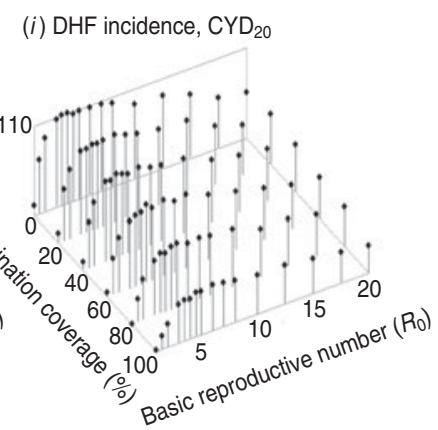

(I) Vaccine-derived DHF, $\mathrm{CYD}_{20}$

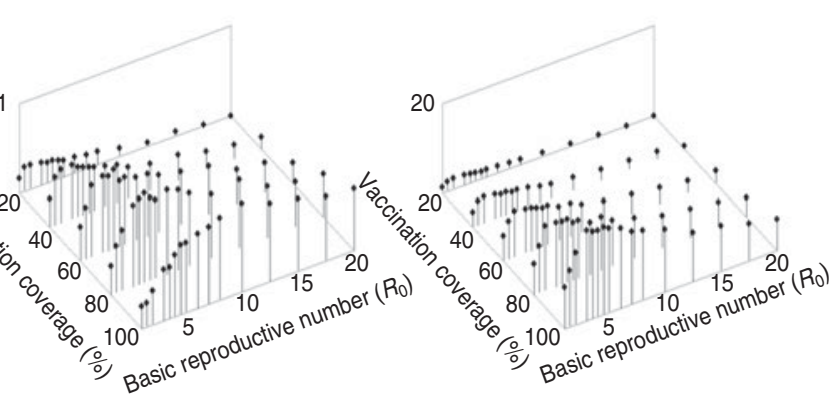

Fig. 3. Results from simulations plotted over mosquito abundance and vaccination coverage. Mosquito abundance is represented as basic reproductive number $\left(R_{0}\right)$. Vaccination coverage is defined as $V$ in the Methods section. $(a-c)$ Viral inoculation rate (/1000 individuals per year), incidence (/100000 individuals per year) of dengue fever (DF) $(d-f)$, dengue haemorrhagic (DHF) $(g-i)$, and DHF attributable to vaccine-derived antibody-dependent enhancement $(j-l)$, were averaged from the last 30 years in each 150-year simulation and then averaged from 20 simulations. $(a, d, g, j)$ $\mathrm{CYD}_{100},(b, e, h, k) \mathrm{CYD}_{40}$, and $(c, f, i, l) \mathrm{CYD}_{20}$ are compared. Parameter setting for the simulations was (TFR, $\left.I, S\right)=$ $(2,0,0 \cdot 2)$. 
(a) $R_{0}=4$

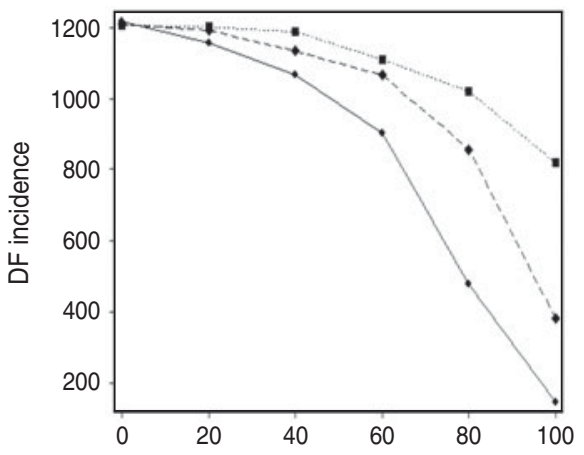

(c) $R_{0}=4$

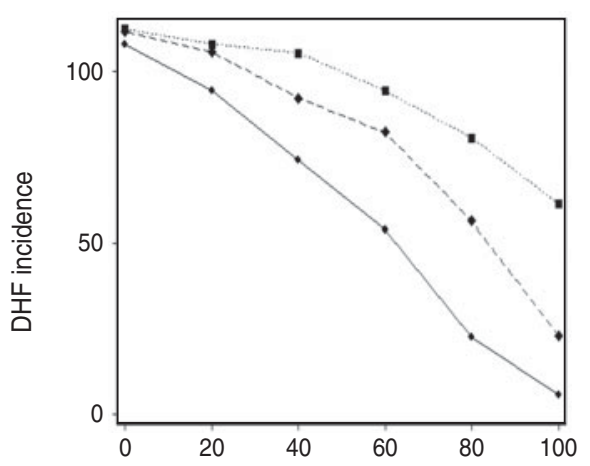

(e) $R_{0}=4$
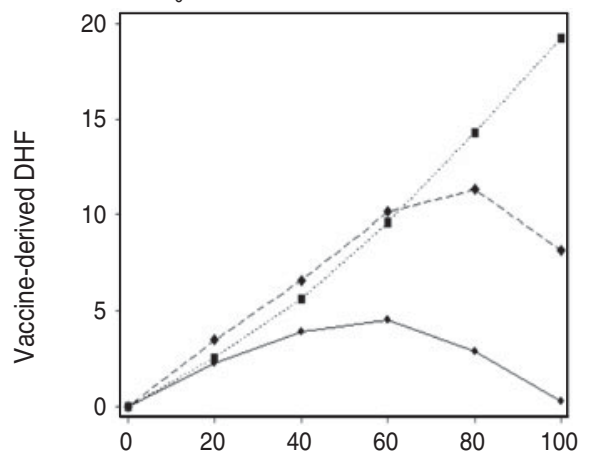

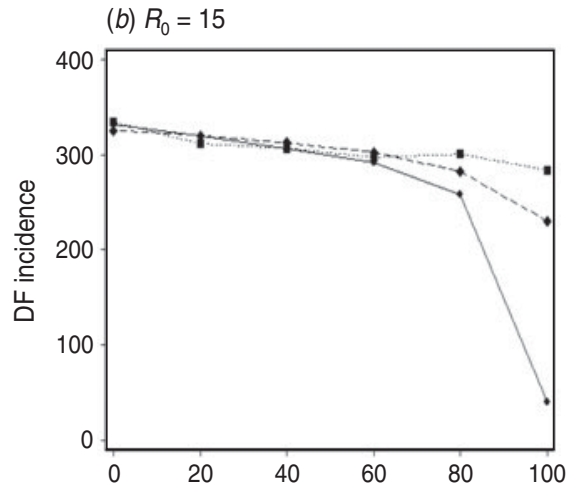

(d) $R_{0}=15$

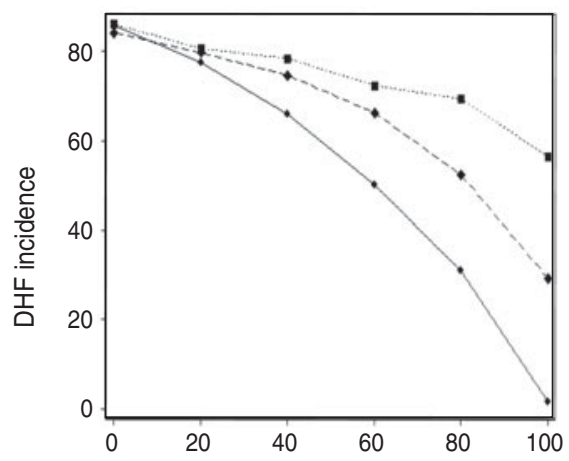

(f) $R_{0}=15$

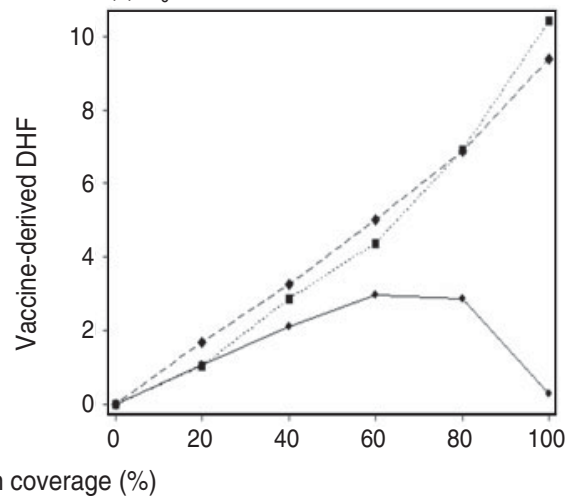

$\longrightarrow \mathrm{CYD}_{100}--\bullet--\mathrm{CYD}_{40} \quad \cdots \cdots \mathbf{\cdots} \cdot \mathrm{CYD}_{20}$

Fig. 4. Incidence of dengue fever (DF), dengue haemorrhagic (DHF), and DHF attributable to vaccine-derived antibodydependent enhancement (ADE), from simulations that assumed moderate or high mosquito abundance, plotted over vaccination coverage. Incidence (/100000 individuals per year) of $(a, b) \mathrm{DF},(c, d) \mathrm{DHF}$, and $(e, f) \mathrm{DHF}$ attributable to vaccinederived $\mathrm{ADE}$, were compared in $\mathrm{CYD}_{100}, \mathrm{CYD}_{40}$, and $\mathrm{CYD}_{20}$. Simulations were conducted assuming $(a, c, e)$ moderate mosquito abundance $\left(R_{0}=4\right)$ or $(b, d, f)$ high mosquito abundance $\left(R_{0}=15\right)$. Parameters were set to the same values as in Figure 3.

used as the initial population structure. Population growth was represented by the total fertility rate (TFR) [43]. Based on the TFR given to a simulation, age-specific birth rate was reconstructed for the each age class of females (Supplementary Appendix 2). The age-specific mortality rate reported from Thailand in 2005 was used throughout the entire simulation. TFR of Thailand was 1.86 in 2000.

\section{Source code}

The source code, which was written in PERL language, can be obtained from Supplementary Appendix 3. 
(a) Viral inoculation rate and I

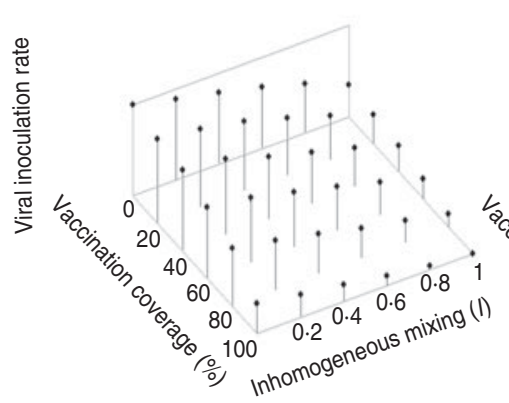

(d) DF incidence and I
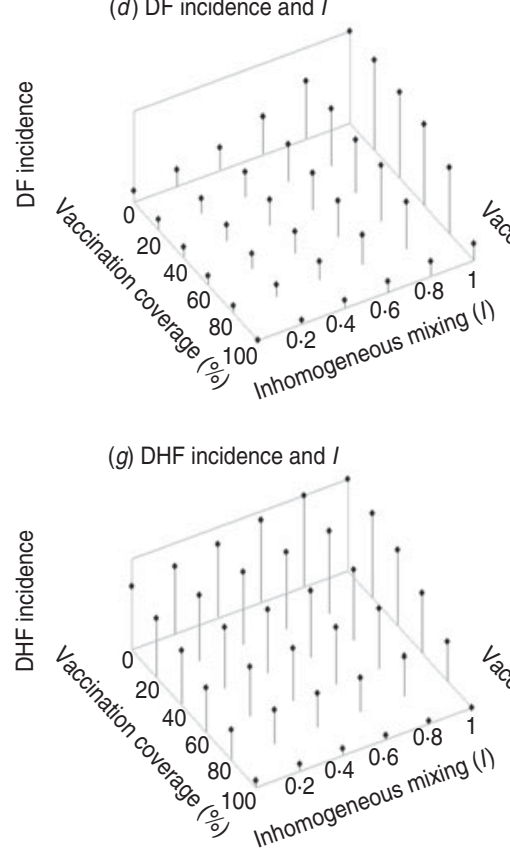

(b) Viral inoculation rate and $S$

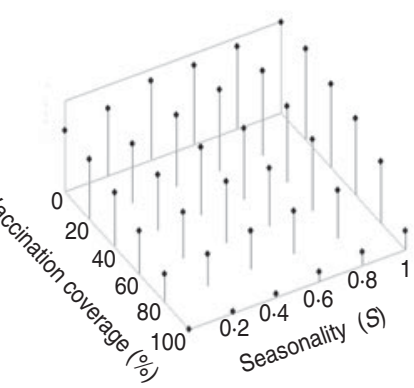

(e) DF incidence and $S$

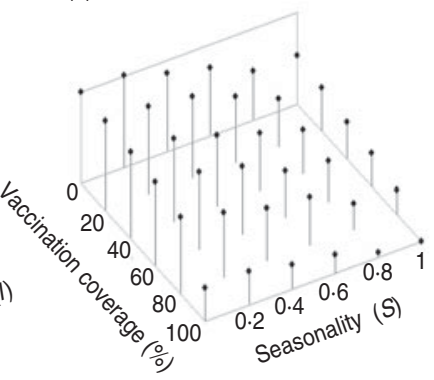

(h) DHF incidence and $S$ (c) Viral inoculation rate and TFR

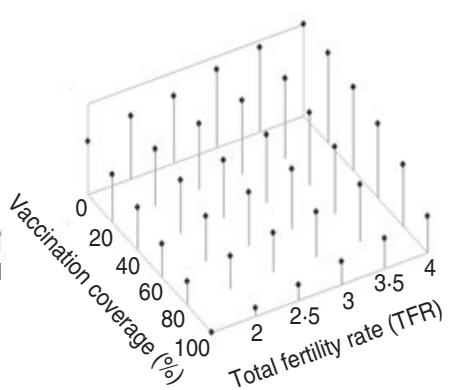

.

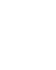



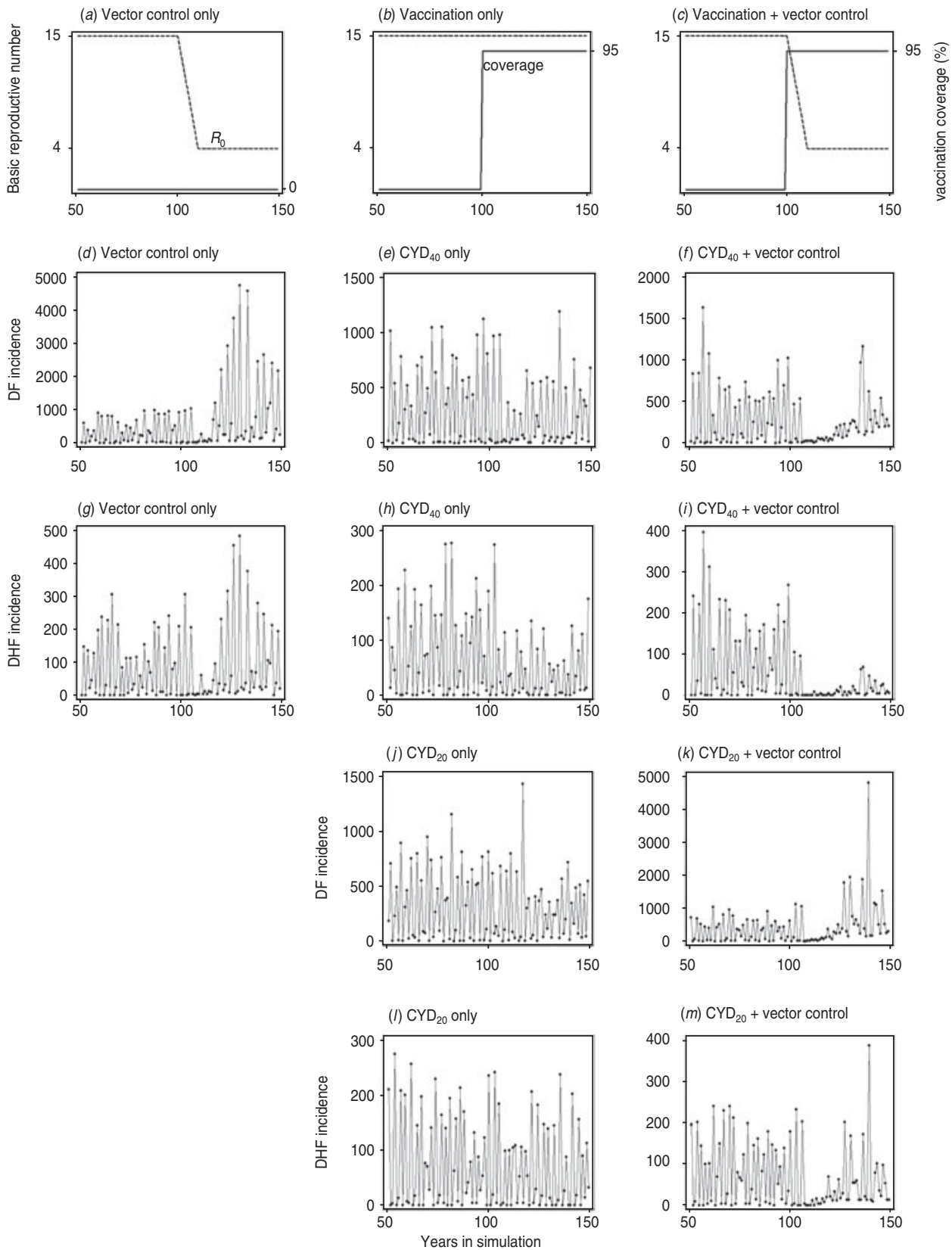

Fig. 6. Temporal patterns in simulations employing different dengue control strategies. Incidence of dengue fever (DF) and dengue haemorrhagic (DHF) is plotted over years in simulation. The strategy was based on either $(a, d, g)$ vector mosquito control only $(b, e, h, j, l)$ vaccination only, or $(c, f, i, k, m)$ vaccination followed by vector control. The temporal change in mosquito abundance $\left(R_{0}\right)$ and vaccination coverage are presented in panels $(a-c)$, while incidence $(/ 100000$ individuals per year) is presented in $(d-f, j, k)$ for DF and in $(g-i, l, m)$ for DHF. CYD 40 was used in $(e, f, h, i)$ while $\mathrm{CYD}_{20}$ was used in $(j-l, m)$. Parameters were set to the same values as in Figure 3.

vaccination on effective reproductive number is presented in Supplementary Appendix 4.

\section{Preventive and predisposing effects of vaccines}

Figure 3 was intersected at $R_{0}=4$ (moderate mosquito abundance) and $R_{0}=15$ (high mosquito abundance;
Fig. 4). The incidence of DF responded to vaccination coverage differently between high and moderate mosquito abundances: DF incidence was more refractory to vaccination in areas of high mosquito abundance (Fig. 4b) than in areas of moderate mosquito abundance (Fig. 4a). The vaccines affected DHF incidence in a contrasting manner. At both high and moderate 
mosquito abundance, all the vaccines reduced DHF incidence (Fig. 4c, $d$ ). Vaccine-derived DHF (Fig. 4e,f) was much less frequent than DHF preventable by $C_{100}$ and $C_{40}$ (Fig. $4 c, d$ ), while this margin was more obscure for $\mathrm{CYD}_{20}$.

\section{Effect of inhomogeneous mixing, seasonality, and population growth}

The effects of inhomogeneous mixing, seasonality, and population growth rate on the epidemiological parameters were examined at different levels of vaccination coverage (Fig. 5). It is intuitive that less inhomogeneous mixing and larger population growth were associated with increased viral inoculation rate (Fig. 5a, c). Interestingly, stronger seasonality was correlated with slightly increased viral inoculation rate (Fig. 5b). However, the response of disease incidence to increased viral inoculation rate was counterintuitive. Under the situation of high mosquito abundance $\left(R_{0}=15\right)$, the increased viral inoculation rate led to decreased DF incidence (Fig. $5 d-f$ ), as predicted by the endemic stability hypothesis. The incidence of DHF and its relationship to vaccination coverage was affected only slightly by the variation in inhomogeneous mixing, seasonality, and population growth rate (Fig. $5 g-i$ ).

\section{Temporal patterns}

In Figure 6, the temporal patterns in the incidence of DF and DHF are presented for three control strategies: mosquito vector control only (Fig. 6a), vaccination only (Fig. 6b), and vaccination followed by vector control (Fig. 6c). In the area of moderate mosquito abundance, all three strategies reduced the incidence of both DF and DHF (data not shown). In contrast, in an area of high mosquito abundance $\left(R_{0}=15\right)$, mosquito control of moderate achievement (to $R_{0}=4$ ) led to an increase in incidence of DF (Fig. 6d) and DHF (Fig. 6g). Vaccination alone by $\mathrm{CYD}_{40}$ did not affect DF incidence markedly (Fig. 6e), but reduced DHF incidence noticeably (Fig. 6h). Vaccination using $\mathrm{CYD}_{40}$ followed by vector control achieved a substantial reduction in incidence of both DF (Fig. 6f) and DHF (Fig. 6i). Vaccination alone by $\mathrm{CYD}_{20}$ did not exert a noticeable influence on the incidence of DF (Fig. 6j) or DHF (Fig. 6l). However, vaccination by $\mathrm{CYD}_{20}$ which preceded vector control attenuated the potential increase in incidence that would have resulted from
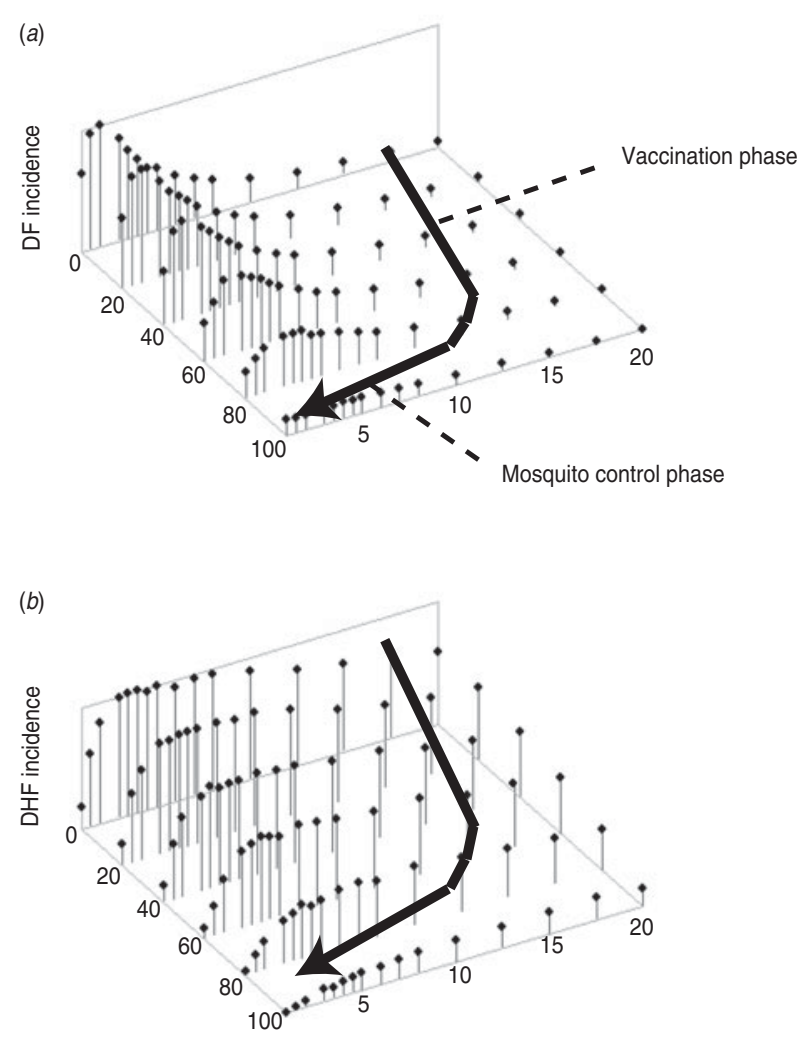

Fig. 7. Optimal strategy to reduce viral inoculation rate and incidence of dengue fever (DF) and dengue haemorrhagic (DHF). The optimal strategy, which reduces the viral inoculation rate and incidence of $\mathrm{DF}$ and $\mathrm{DHF}$, is superimposed on the results of simulations which used $\mathrm{CYD}_{40}$ (Fig. 3). This strategy, which is represented by the curved arrow in $(a)$ and $(b)$ is composed of a vaccination phase, and a mosquito control phase. Initially, by attaining high coverage of vaccination in 'vaccination phase', (a) DF incidence and (b) DHF incidence decrease. In the subsequent 'mosquito control phase', $R_{0}$ is reduced, thereby decreasing these incidences to a lower level. With this strategy, combining vaccination and mosquito control, the ridges of incidence of $(a) \mathrm{DF}$ and $(b)$ DHF can be circumvented.

vector control alone for DF (cf. Fig. $6 d, k$ ) and DHF (cf. Fig. $6 g, m$ ).

\section{DISCUSSION}

Individual-based model simulation has become increasingly common for comparing disease control strategies [44-46]. The present study employed this methodology to predict the effects of vector control and vaccination on the incidence of dengue-related diseases. 
As a result, it was predicted that mosquito control alone is likely to increase the incidence of both DF and DHF in areas of high mosquito abundance. Although extremely strong suppression of vector abundance could decrease the incidence eventually, incidence would increase transiently while mosquito reduction remains incomplete. Despite this concern, mosquito control activities will probably be continued in developing countries, partly because being endemic for dengue virus reduces the attractiveness for tourism and overseas investment. Since the vector mosquito larvae infest intended water containers as well as disposals [47, 48], not only mosquito control but also improvement in water supply and garbage collection systems will decrease mosquito abundance $[49,50]$.

The present study emulated seroconversion rates of CYD, the dengue vaccine with the highest prospect of proceeding to phase III trial. Sensitivity analyses were conducted to compensate for the uncertainties in seroconversion rates. Consequently, the main conclusion was not affected by these uncertainties; the vaccine could attenuate the possible surge in DF and DHF driven by the decrease in mosquito abundance. However, the predisposing effect of vaccine-derived ADE would vary greatly depending on protective seroconversion rates. It should be noted that the leastfavourable assumptions for a vaccine were adopted, including that vaccines always induce enhancing antibodies, the enhancing capability persists throughout life, and the vaccine does not confer cross-protection. Hence, the estimates of a predisposing effect of vaccine may be exaggerated. In addition, DHF cases caused by vaccine-derived ADE would be much fewer than DHF cases prevented by a vaccine of considerably high (although not perfect) seroconversion rates. Therefore, a vaccination programme which uses such a sub-optimal vaccine may be justifiable, at least in areas of high mosquito abundance where mosquito reduction may increase the incidence of DF and DHF. As represented by the arrows in Figure 7, the peaks of DF/DHF incidence could be circumvented if mosquito control is preceded by high vaccination coverage. Since some may feel that any vaccine that predisposes vaccinees to the risk of DHF cannot be ethically acceptable, the present study provides quantitative information to ethical and economic discussions of this issue. Although vaccination of not only small children but a large part of the population may be necessary at the initial phase of a vaccination programme [51], the present study did not investigate this important topic. Further studies are warranted on these issues.

Collectively, the present study proposes a new methodology to predict and compare the populationlevel effect of dengue vaccines. The prediction can be updated easily as seroconversion rates are improved, or as currently unknown parameters are reported from field/experimental studies. The predictions made here, however peculiar they may appear, should be considered in developing a global dengue control strategy.

\section{ACKNOWLEDGEMENTS}

The authors thank Katia Koelle, Eiji Konishi, Bruno Guy, Paul G. Coleman, and the late Clive Davies for assistance and advice. The corresponding author would be pleased to provide technical assistance in installing, executing, and modifying the simulation software upon request.

\section{SUPPLEMENTARY MATERIAL}

For supplementary material accompanying this paper visit http://dx.doi.org/10.1017/S0950268813001866.

\section{DECLARATION OF INTEREST}

None.

\section{REFERENCES}

1. Kalayanarooj S. Standardized clinical management: evidence of reduction of dengue haemorrhagic fever case-fatality rate in Thailand. Dengue Bulletin 1999; 23: $10-17$.

2. WHO Regional Office for South-East Asia. Dengue/ DHF: case fatality rate $(\%)$ of DF/DHF in the SouthEast Asia Region (1985-2006). (http://www.searo. who.int/en/Section10/Section332_1102.htm). Accessed 24 September 2007.

3. Anderson RM, May RM. Infectious Diseases of Humans: Dynamics and Control. New York: Oxford University Press, 1991.

4. Dantes HG, et al. Dengue epidemics on the Pacific Coast of Mexico. International Journal of Epidemiology 1988; 17: 178-186.

5. Egger JR, Coleman PG. Age and clinical dengue illness. Emerging Infectious Diseases 2007; 13: 924-925.

6. Egger JR, et al. Reconstructing historical changes in the force of infection of dengue fever in Singapore: implications for surveillance and control. Bulletin of the World Health Organization 2008; 86: 187-196. 
7. Guha-Sapir D, Schimmer B. Dengue fever: new paradigms for a changing epidemiology. Emerging Themes in Epidemiology 2005; 2: 1.

8. Coleman PG, Perry BD, Woolhouse ME. Endemic stability - a veterinary idea applied to human public health. Lancet 2001; 357: 1284-1286.

9. Nagao Y, Koelle K. Decreases in dengue transmission may act to increase the incidence of dengue hemorrhagic fever. Proceedings of the National Academy of Sciences USA 2008; 105: 2238-2243.

10. Halstead SB, Shotwell H, Casals J. Studies on the pathogenesis of dengue infection in monkeys. II. Clinical laboratory responses to heterologous infection. Journal of Infectious Diseases 1973; 128: 15-22.

11. Sabin AB. Research on dengue during World War II. American Journal of Tropical Medicine and Hygiene 1952; 1: 30-50.

12. Sangkawibha $\mathbf{N}$, et al. Risk factors in dengue shock syndrome: a prospective epidemiologic study in Rayong, Thailand. I. The 1980 outbreak. American Journal of Epidemiology 1984; 120: 653-669.

13. Thammapalo $\mathbf{S}$, et al. Relationship between transmission intensity and incidence of dengue hemorrhagic fever in Thailand. PLoS Neglected Tropical Diseases 2008; 2: e263.

14. Sun W, et al. Phase 2 clinical trial of three formulations of tetravalent live-attenuated dengue vaccine in flavivirusnaive adults. Human Vaccines 2009; 5: 33-40.

15. Guy B, et al. From research to phase III: preclinical, industrial and clinical development of the Sanofi Pasteur tetravalent dengue vaccine. Vaccine 2011; 29: 7229-7241.

16. Sabchareon A, et al. Protective efficacy of the recombinant, live-attenuated, CYD tetravalent dengue vaccine in Thai schoolchildren: a randomised, controlled phase 2b trial. Lancet 2012; 380: 1559-1567.

17. Morens DM. Antibody-dependent enhancement of infection and the pathogenesis of viral disease. Clinical Infectious Diseases 1994; 19: 500-512.

18. Huisman W, et al. Vaccine-induced enhancement of viral infections. Vaccine 2009; 27: 505-512.

19. Guy B, et al. Evaluation by flow cytometry of antibodydependent enhancement (ADE) of dengue infection by sera from Thai children immunized with a liveattenuated tetravalent dengue vaccine. Vaccine 2004; 22: 3563-3574.

20. Poo J, et al. Live-attenuated tetravalent dengue vaccine in dengue-naive children, adolescents, and adults in Mexico City: randomized controlled phase 1 trial of safety and immunogenicity. Pediatric Infectious Disease Journal 2011; 30: e9-e17.

21. Morrison D, et al. A novel tetravalent dengue vaccine is well tolerated and immunogenic against all 4 serotypes in flavivirus-naive adults. Journal of Infectious Diseases 2010; 201: 370-377.

22. Beatty M, et al. Assessing the potential of a candidate dengue vaccine with mathematical modeling. PLoS Neglected Tropical Diseases 2012; 6: e1450.

23. Grimm V, et al. Pattern-oriented modeling of agentbased complex systems: lessons from ecology. Science 2005; 310: 987-991.
24. Kliks SC, et al. Evidence that maternal dengue antibodies are important in the development of dengue hemorrhagic fever in infants. American Journal of Tropical Medicine and Hygiene 1988; 38: 411-419.

25. Brandt WE, et al. Infection enhancement of dengue type 2 virus in the U-937 human monocyte cell line by antibodies to flavivirus cross-reactive determinants. Infection and Immunity 1982; 36: 1036-1041.

26. Morens DM, Halstead SB, Marchette NJ. Profiles of antibody-dependent enhancement of dengue virus type 2 infection. Microbial Pathogenesis 1987; 3: 231237.

27. Guirakhoo F, et al. Live attenuated chimeric yellow fever dengue type 2 (ChimeriVax-DEN2) vaccine: Phase I clinical trial for safety and immunogenicity: effect of yellow fever pre-immunity in induction of cross neutralizing antibody responses to all 4 dengue serotypes. Human Vaccines 2006; 2: 60-67.

28. Anderson KB, et al. A short time interval between first and second dengue infections is associated with protection from clinical illness in a prospective schoolbased cohort in Thailand. In: Annual Meeting of the American Society of Tropical Medicine and Hygiene, Atlanta, GA, USA, 2012.

29. Guzman MG, et al. Epidemiologic studies on dengue in Santiago de Cuba, 1997. American Journal of Epidemiology 2000; 152: 793-799; discussion 804.

30. Gonzalez D, et al. Classical dengue hemorrhagic fever resulting from two dengue infections spaced 20 years or more apart: Havana, Dengue 3 epidemic, 20012002. International Journal of Infectious Diseases 2005; 9: 280-285.

31. Vaughn DW, et al. Dengue viremia titer, antibody response pattern, and virus serotype correlate with disease severity. Journal of Infectious Diseases 2000; 181: 2-9.

32. Wearing HJ, Rohani P. Ecological and immunological determinants of dengue epidemics. Proceedings of the National Academy of Sciences USA 2006; 103: 11802 11807.

33. Chikaki E, Ishikawa H. A dengue transmission model in Thailand considering sequential infections with all four serotypes. Journal of Infection in Developing Countries 2009; 3: 711-722.

34. Massad E, et al. Dengue and the risk of urban yellow fever reintroduction in Sao Paulo State, Brazil. Revista de Saude Publica 2003; 37: 477-484.

35. Coelho GE, et al. Dynamics of the 2006/2007 dengue outbreak in Brazil. Memorias do Instituto Oswaldo Cruz 2008; 103: 535-539.

36. Macdonald G. The analysis of equilibrium in malaria. Tropical Diseases Bulletin 1952; 49: 813-829.

37. Macdonald G. The Epidemiology and Control of Malaria. Oxford: Oxford University Press, 1957.

38. Ferguson NM, Donnelly CA, Anderson RM. Transmission dynamics and epidemiology of dengue: insights from age-stratified sero-prevalence surveys. Philosophical Transactions of the Royal Society of London, Series B: Biological Sciences 1999; 354: 757-768. 
39. Johansson MA, Hombach J, Cummings DA. Models of the impact of dengue vaccines: a review of current research and potential approaches. Vaccine 2011; 29: 5860-5868.

40. Gubler DJ, et al. Viraemia in patients with naturally acquired dengue infection. Bulletin of the World Health Organization 1981; 59: 623-630.

41. Hombach J, et al. Report on a WHO consultation on immunological endpoints for evaluation of new Japanese encephalitis vaccines, WHO, Geneva, 2-3 September, 2004. Vaccine 2005; 23: 5205-5211.

42. Strickman D, Kittayapong P. Dengue and its vectors in Thailand: introduction to the study and seasonal distribution of Aedes larvae. American Journal of Tropical Medicine and Hygiene 2002; 67: 247-259.

43. Russel J, Cohn R. Total Fertility Rate. Edinburgh: Lennex Corp, 2012.

44. Epstein JM, et al. Toward a Containment Strategy for Smallpox Bioterror: An Individual-based Computational Approach. Washington, D.C.: Brookings Institution Press, 2004.

45. Vardavas R, Breban R, Blower S. Can influenza epidemics be prevented by voluntary vaccination? PLoS Computational Biology 2007; 3: e85.
46. Morimoto T, Ishikawa H. Assessment of intervention strategies against a novel influenza epidemic using an individual-based model. Environmental Health and Preventive Medicine 2010; 15: 151-161.

47. Focks DA, Chadee DD. Pupal survey: an epidemiologically significant surveillance method for Aedes aegypti: an example using data from Trinidad. American Journal of Tropical Medicine and Hygiene 1997; 56: 159-167.

48. Manrique-Saide P, et al. Pupal surveys for Aedes aegypti surveillance and potential targeted control in residential areas of Merida, Mexico. Journal of the American Mosquito Control Association 2008; 24: 289-298.

49. Knudsen AB, Slooff R. Vector-borne disease problems in rapid urbanization: new approaches to vector control. Bulletin of the World Health Organization 1992; 70: 1-6.

50. Reiter P, Gubler DJ. Surveillance and control of urban dengue vectors. In: Gubler DJ, Kuno G, eds. Dengue and Dengue Hemorrhagic Fever. Wallingford: CAB International, 1998, pp. 425-462.

51. Massad E, et al. A model-based design of a vaccination strategy against rubella in a non-immunized community of Sao Paulo State, Brazil. Epidemiology and Infection 1994; 112: 579-594. 